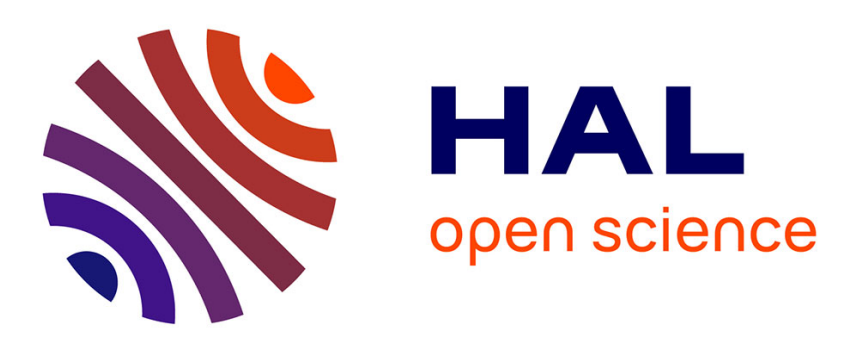

\title{
La sous-traitance comme moyen de subordination réelle de la force de travail
}

\author{
Bruno Tinel, Corinne Perraudin, Nadine Thevenot, Julie Valentin
}

\section{To cite this version:}

Bruno Tinel, Corinne Perraudin, Nadine Thevenot, Julie Valentin. La sous-traitance comme moyen de subordination réelle de la force de travail. Actuel Marx, 2007, premier semestre (41), pp.153-164. halshs-00266368

\section{HAL Id: halshs-00266368 \\ https://shs.hal.science/halshs-00266368}

Submitted on 21 Mar 2008

HAL is a multi-disciplinary open access archive for the deposit and dissemination of scientific research documents, whether they are published or not. The documents may come from teaching and research institutions in France or abroad, or from public or private research centers.
L'archive ouverte pluridisciplinaire $\mathbf{H A L}$, est destinée au dépôt et à la diffusion de documents scientifiques de niveau recherche, publiés ou non, émanant des établissements d'enseignement et de recherche français ou étrangers, des laboratoires publics ou privés. 


\title{
La sous-traitance comme moyen de subordination réelle de la force de travail
}

\author{
Bruno Tinel, Corinne Perraudin, Nadine Thèvenot, Julie Valentin
}

La généralisation des pratiques de sous-traitance à laquelle nous assistons en France depuis une vingtaine d'années représente un moment historique particulier dans l'histoire de la subordination de la force de travail au capital ${ }^{1}$. Certes, ce type de pratiques est ancien mais, en la matière, une nouveauté se doit d'être expliquée : les données dont nous disposons pour la France montrent que le recours à la sous-traitance a connu un essor considérable depuis vingt ans. En 2003, 9 entreprises industrielles sur 10 sont donneurs d'ordres et leur taux de sous-traitance, en moyenne, a plus que doublé sur cette période ${ }^{2}$.

En moyenne, pour l'année 2003, les donneurs d'ordres ont une masse salariale totale répartie à concurrence de $88,8 \%$ pour leurs salariés en interne et $11,2 \%$ pour les travailleurs externes mobilisés via la sous-traitance (voir graphique 2 en annexe). Or, cette évaluation est obtenue par une méthode qui donne uniquement un niveau minimal de la part des dépenses de main-d'œuvre dans les dépenses de sous-traitance. De plus, comme les salaires sont plus faibles dans les entreprises preneurs d'ordres que dans les entreprises donneurs d'ordres ${ }^{3}$, le contenu en emploi d'un même montant de masse salariale est plus élevé pour les preneurs d'ordres que pour les donneurs d'ordres. Ainsi, plus de $10 \%$ des travailleurs de l'industrie sont extériorisés par les donneurs d'ordres. Ils peuvent dépendre d'une convention collective moins protectrice et moins avantageuse que celle s'appliquant aux donneurs d'ordres ${ }^{4}$. Les conséquences portent également sur des conditions d'emploi et de travail moins favorables que celles dont bénéficient les travailleurs employés dans des entreprises non-preneurs d'ordres, en termes de salaires et d'éclatement de la relation de travail. Enfin, ils ne bénéficient pas d'un contrat de travail avec l'employeur qui dirige réellement leur travail et qui se trouve responsable, en fait et en droit, de leur emploi.

Pour expliquer ce phénomène, il est très fréquent d'évoquer l'instabilité croissante de l'environnement économique dans lequel les entreprises évolueraient aujourd'hui. Pourtant, les calculs que nous avons effectués ne permettent pas de donner un crédit important à cette thèse : les entreprises substituent de manière croissante une relation commerciale à du travail direct, mais ceci ne résulte pas d'un besoin accru de flexibilité dans la gestion de la main-d'œuvre qui découlerait des fluctuations de l'activité ${ }^{5}$.

\footnotetext{
Nous avons proposé une première interprétation de ce phénomène dans un article (N. Thèvenot, B. Tinel, J. Valentin, «État social et sous-traitance. Quelles transformations des dépendances dans le travail ? ») présenté au colloque État et Régulation Sociale. Comment penser la cohérence de l'intervention publique?, 11-13 sept. 2006 à INHA, organisé par le Matisse.

2 Voir graphique 1 en annexe. Pour une description statistique du phénomène à partir d'une base de données construite sur les Enquêtes Annuelles d'Entreprises sur la période 1984-2003 et regroupant les entreprises industrielles de 20 salariés et plus, cf. C. Perraudin, N. Thèvenot, J. Valentin, «Sous-traiter ou embaucher ? Une analyse empirique des comportements de substitution des entreprises de l'industrie en France entre 1984 et 2003 », Document de travail CEE, présenté aux XIII ${ }^{\text {èmes }}$ journées d'études sur les données longitudinales dans l'analyse du marché du travail, LEST-1 ${ }^{\mathrm{er}}-2$ juin 2006; N. Thèvenot, J. Valentin, « La sous-traitance comme alternative au contrat de travail : une évaluation empirique pour la France, 1984-2000», Economie Appliquée, tome LVIII, n`3, 2005, pp. 51-79 et J. Valentin (dir.), , «Relations de travail et dynamiques des arbitrages des firmes vis-à-vis des dispositifs juridiques : quelles substitutions ? Le recours aux CDD et à la sous-traitance en France 1984-2000», Rapport du Matisse - CES pour le Ministère de l'emploi et de la solidarité - DARES, Mission Animation de la recherche, Avril 2006.

3 Dans l'échantillon, en 2003, la moyenne de la rémunération brute annuelle par salarié est égale à 35063 euros dans les entreprises donneurs d'ordres, contre 31709 euros dans les entreprises preneurs d'ordres.

4 Voir notamment les exemples donnés par J. Le Goff (Du silence à la parole : une histoire du droit du travail en France de 1830 à nos jours, Rennes, Presses Universitaires de Rennes, 2004) d'entreprises sous-traitant pour transférer une partie de leur activité dans des branches professionnelles caractérisées par des conventions collectives moins protectrices. Sur les inégalités entre conventions collectives et l'émiettement des branches professionnelles, voir A. Jobert, Les Espaces de la négociation collective, branches et territoires, Toulouse, Octares Editions, 2000.

5 Tous les calculs dont il est question dans cet article ont été réalisés à partir des Enquêtes Annuelles d'Entreprises qui
} 
La sous-traitance n'est pas nouvelle, et elle est classiquement interprétée soit comme une soustraitance de spécialité, soit comme une sous-traitance de capacité. Dans le premier cas, l'entreprise donneur d'ordres ne dispose pas en interne des compétences particulières requises pour répondre à sa croissance ou aux transformations de l'organisation du travail, et cette ressource n'est pas offerte sous forme standardisée. Elle fait alors appel à une entité extérieure capable de produire pour elle du «sur mesure ». Dans le cas de la sous-traitance de capacité ${ }^{6}$, où une entreprise achète un bien ou un service qu'elle produit déjà, l'externalisation a lieu parce que l'entreprise peut acheter à une autre entreprise ce bien ou service dont la production requiert des compétences non spécifiques pour un prix plus faible. L'offreur est compétitif car son échelle de production est plus importante que celle du demandeur et il est plus avancé que lui dans la subordination réelle de sa main-d'œuvre sur ce segment de production. La standardisation est alors permise par l'existence de débouchés importants dans les autres branches de l'économie. Ces deux explications, fréquemment avancées pour expliquer pourquoi une entreprise sous-traite certaines activités à une autre entreprise, ne permettent pas de rendre compte de l'essor considérable de ce phénomène en France depuis vingt ans. À moins de céder à un certain technologisme qui nous ramènerait à la première explication, invalidée par nos tests économétriques, on ne voit pas pourquoi les entreprises auraient subitement eu un besoin accru de sous-traitance de spécialité et de capacité. Ces éléments jouent sans doute aujourd'hui, mais pas davantage qu'il y a trente ou quarante ans.

Nous proposons une autre explication de l'essor de la sous-traitance à partir des notions de subordination formelle et réelle élaborées par Marx dans le Chapitre inédit du Capital. Au regard de l'histoire de l'économie française, la montée récente de la sous-traitance traduirait un moment bien particulier de l'histoire de la subordination de la main-d'œuvre au capital : elle constituerait l'une des modalités par lesquelles celui-ci cherche à contourner un certain nombre de protections instituées de haute lutte par le salariat. Il s'agirait, dès lors, non pas d'un phénomène nécessaire en soi pour répondre à des exigences imposées également à tous les acteurs en présence par la «nature » économique, mais d'une modalité très contemporaine de la lutte des classes, mettant en jeu des intérêts identifiables et antagoniques.

En préalable à l'analyse de la sous-traitance, il est utile de rappeler le contenu des notions que nous utilisons $^{7}$. Pour Marx, dans les premiers temps du capitalisme, la séparation entre le producteur et les moyens de production conduit, dans le procès de travail, à la subordination formelle d'une force de travail, jusque-là artisanale. Dans un second temps, le procès de production lui-même fait l'objet d'une recomposition, notamment au moyen du machinisme, qui subordonne désormais réellement la force de travail aux objectifs de valorisation du capital. La subordination réelle s'est approfondie et généralisée, au $\mathrm{XX}^{\mathrm{e}}$ siècle, par l'essor de la grande entreprise en lien avec la montée de l'État social $^{8}$. Dès que le rapport de force est devenu plus favorable, le capital a chercher contourner les protections salariales mises en place sous le fordisme. La sous-traitance se présente comme l'une des modalités de ce contournement dont la logique consiste à faire éclater formellement le collectif de travail tout en maintenant l'unité réelle du capital, malgré une fragmentation apparente.

rendent compte, en particulier, des comportements de sous-traitance de l'ensemble des entreprises de 20 salariés et plus. Nous travaillons ici sur un champ restreint aux entreprises de l'industrie, des biens d'équipement, des biens intermédiaires et des biens de consommation. Voir C. Perraudin, N. Thèvenot, B. Tinel, J. Valentin, « Sous-traitance et ineffectivité du droit du travail : une analyse économique », miméo, 2006.

6 Notons que, d'un point de vue économétrique, il est très difficile de dissocier capacité et instabilité, car on teste la capacité par un indicateur d'incertitude.

7 Pour une synthèse de l'analyse par Marx du processus de production que nous faisons nôtre, voir B. Tinel « Marx, Organisation et exploitation du travail », Cahiers de la MSE, Série Rouge, R05072, nov. 2005, dont nous reprenons ici le point 7 ; nous avons utilisé les références suivantes de Marx : Un chapitre inédit du Capital, Paris, UGE (10/18), 1971 et Le Capital in Euvres Economie I, Paris, Gallimard coll. La Pléiade, 1965.

8 Sur la notion d'État Social, cf. C. Ramaux, Emploi : éloge de la stabilité, L'État social contre la flexicurité, Paris, Mille et une nuits, 2006. 


\section{De la subordination formelle à la subordination réelle du travail au capital}

Dans un texte resté longtemps inédit, connu sous le nom de $V I^{e}$ chapitre inédit $d u$ Capital, Marx approfondit l'analyse de la subordination, ou de la soumission, du travail au capital. Il distingue la soumission formelle de la soumission réelle. La soumission formelle se rapporte aux débuts du capitalisme, moment où le capital s'empare d'un procès de travail encore artisanal, qu'il ne maîtrise donc pas encore ; mais elle continue par la suite à coexister comme forme particulière au sein du mode de production capitaliste pleinement développé.

Dans les débuts de la manufacture, le capital ne change rien au mode de production réel, il se contente de rassembler des artisans de métier. La subordination du procès de travail se traduit uniquement par le fait que "l'ouvrier passe sous le commandement, la direction et la surveillance $d u$ capitaliste $»^{9}$. Le capital n'a alors pas de prise sur le contenu du travail, il se contente d'agir par la surveillance sur la quantité de travail. Par rapport au système artisanal, où l'ouvrier est alternativement producteur puis vendeur de son produit, la soumission formelle rend continu le procès de travail car le producteur, devenu salarié, n'a plus à s'interrompre pour vendre les marchandises. Le travailleur vend sa force de travail au capitaliste et c'est ce dernier qui, désormais, vend le produit du travail. Cette séparation entre le travailleur et son produit, où la tâche de production incombe au salarié et celle de vente au capitaliste, est en quelque sorte la forme primitive de la division capitaliste du travail. Autrement dit, l'ouvrier artisan devient salarié parce qu'il vend désormais sa force de travail au lieu du produit de son travail. Le premier moment de la production capitaliste correspond donc à la prise de contrôle de la marchandise «force de travail » par le capital. Cette prise de contrôle « libère » l'ouvrier de la tâche de commercialisation et permet ainsi au capital d'exiger, par la surveillance et le contrôle, un effort productif continu. Réduire les porosités de la journée de travail et accroître sa durée totale, c'est-à-dire augmenter l'intensité et le temps de travail par la coercition, est le seul moyen pour le capital d'obtenir et d'accroître la plusvalue. La production de «plus-value absolue» se présente donc comme spécifique à la subordination formelle.

Une fois la soumission formelle du travail acquise par le capital, celui-ci s'empare progressivement du procès de production et le transforme de manière à ce qu'il réponde au besoin de sa propre valorisation, ce qui donne lieu à un mode de production désormais spécifiquement capitaliste, lequel «révolutionne progressivement la technique du travail et le mode d'existence réel de l'ensemble du procès de travail en même temps que les rapports entre les divers agents de la production $»^{10}$. La soumission réelle du travail au capital correspond donc à l'appropriation, non plus seulement du produit et du temps de travail, mais de la manière même dont s'exécute le travail. Celui-ci devient réellement un instrument du capital. Par la recomposition de la division du travail, tout d'abord dans la manufacture, puis avec le machinisme, le capital parvient à réduire la valeur de la force de travail. La subordination réelle est donc le moyen d'obtenir la « plus-value relative ».

Marx considère que ces deux formes de subordination non seulement coexistent, mais donnent lieu l'une à l'autre. La subordination ne peut devenir réelle que si elle se présente déjà comme formelle. En outre, en transformant le procès de production, elle donne naissance à des branches nouvelles qui, au début de leur développement, se contentent de soumettre formellement la force de travail. Toute nouvelle activité qui émerge pour répondre aux besoins nouveaux du développement de la production spécifiquement capitaliste dans les branches déjà existantes est d'abord plus ou moins artisanale dans son organisation, c'est-à-dire fondée en premier lieu sur les savoir-faire de travailleurs réellement autonomes et formellement soumis au capital. Ce n'est que dans un second temps que celui-ci s'approprie à nouveau ce procès de travail en le recomposant et en le codifiant, bref en le rendant extérieur au travailleur, c'est-à-dire en l'objectivant. À mesure que se développe la production capitaliste, une soumission à la fois formelle et réelle se produit, dans des branches

K. Marx, Un chapitre inédit du Capital, op. cit., p. 176-7.

10 K. Marx, Un chapitre inédit du Capital, op. cit.,, p. 194, souligné par Marx. 
différentes. L'une précède l'autre dans une même branche, puis la seconde suscite la première dans une nouvelle branche, et ainsi de suite.

$\mathrm{Au}$ fil du développement capitaliste, le renouvellement du processus d'appropriation du métier par le capital, au moyen de la soumission formelle puis réelle, opère constamment. De nouvelles branches sont créées par le développement même de la soumission réelle du travail au capital dans les industries les plus développées. Au départ, la dimension modeste de ces branches nouvelles fait que le capital ne peut matériellement pas soumettre d'emblée le travail de manière réelle. Il est contraint de tolérer que le procès de production demeure par son contenu entre les mains de travailleurs très qualifiés. La valeur de cette force de travail experte demeure élevée et le capital n'aura de cesse de la réduire par la soumission réelle à mesure de son développement.

\section{De la subordination réelle à l'essor de la sous-traitance : construction et contournement de l'État social}

Avec le fordisme, qui voit s'étendre la grande entreprise industrielle au cours des «Trente glorieuses », se généralise la subordination réelle de la force de travail. Dans un contexte de pleinemploi, résultant notamment de politiques keynésiennes actives, de luttes et de compromis sociaux mettant en jeu à la fois les forces syndicales à l'intérieur des frontières et la menace soviétique à l'extérieur ${ }^{11}$, cet accroissement considérable de la puissance du capital s'est accompagné d'une montée des protections accordées à la force de travail (sécurité sociale et droit du travail, notamment: comités d'entreprises, négociation collective, reconnaissance du droit syndical, protection contre les licenciements) ${ }^{12}$. L'essor de l'État social soutient et aménage celui de l'organisation industrielle monopoliste et il apporte en même temps des protections et des ressources pour la force de travail ${ }^{13}$. Durant cette période, le capital s'est vu contraint de concéder du terrain au travail dans tous les domaines, en échange de l'approfondissement de la subordination réelle par la réussite même du projet keynésien. Mais, ensuite, à la faveur de l'affaiblissement du pouvoir de négociation du travail résultant de la montée du chômage de masse au cours des années 1970, le capital chercha à contourner la puissance acquise par le salariat dans le cadre de la grande entreprise industrielle.

Initiée par le capital, la sous-traitance est l'une des facettes de ce contournement des compromis institutionnalisés par l'État social. En effet, le donneur d'ordres ne s'engage pas dans une relation de travail directe et il n'a plus affaire au droit du travail, car le recrutement est remplacé formellement par un contrat commercial, tout en mobilisant réellement la main-d'œuvre. La sous-traitance constitue une forme de mobilisation de la main-d'œuvre par laquelle les entreprises donneurs d'ordres recourent à des entités qui dépendent d'elles, non seulement au niveau monétaire, mais aussi en termes de pouvoir de contrôle du procès de travail. Autrement dit, la subordination réelle du travailleur qui jusque-là avait lieu à l'intérieur de l'entreprise se poursuit au-delà de l'entreprise, et entre les entreprises. De ce fait, la sous-traitance contribue à dessiner une hiérarchie entre les unités productives.

Cette double dépendance, caractéristique des situations de sous-traitance dans la période actuelle, transforme le rapport salarial tel qu'il s'est construit dans la période fordiste en remettant en cause (en rendant moins efficaces) les modes par lesquels l'État social avait développé le droit du travail et la protection sociale. Lorsqu'une entreprise fait exécuter une prestation de travail par une personne dans le cadre de la sous-traitance, la relation donneur d'ordres/sous-traitant se définit par le fait que le donneur d'ordres fait exécuter par le sous-traitant un travail que ce dernier n'aurait autrement pas réalisé, et c'est en ce sens que le donneur d'ordres mobilise du travail. Le travailleur

\footnotetext{
11 Voir L. Hoang-Ngoc, B. Tinel, «La régulation du 'nouveau capitalisme'. Analyses positives et recommandations normatives comparées », Economie Appliquée, LVIII, n¹, 2005pp. 33-57.

12 F. Collin, R. Dhoquois, P.-H. Goutierre, A. Jeammaud, G. Lyon-Caen, A. Roudil, Le Droit capitaliste du travail, Grenoble, Presses Universitaires de Grenoble, coll. « Critique du droit », 1980.

13 Il n'est donc pas un simple «État de classe ».
} 
collectif est alors formellement éclaté entre travail directement mobilisé par le capital donneur d'ordres et travail indirect mobilisé sous forme de sous-traitance. Mais la logique de fond régissant la division du travail n'entame pas le processus de subordination réelle. L'autonomie ou l'indépendance en droit du capital preneur d'ordre est illusoire en fait.

\section{Unité et hiérarchisation du capital : la sous-traitance comme moyen de subordination}

Parce qu'elle consiste à mobiliser du travail autrement qu'en ayant recours au travail salarié en interne, la sous-traitance constitue une forme de substitution, de la part de l'entreprise donneur d'ordres, au contrat de travail salarié. Au sens strict, la « sous-traitance de substitution » est motivée par un différentiel de prix entre donneur d'ordres et sous-traitant, qui ne résulte pas ici d'un différentiel de coût de production ou d'un défaut de compétences en tant que tels, mais d'un contournement des conditions d'emploi qui prévalent dans l'entreprise donneur d'ordres. Cependant, même lorsque les motifs de la sous-traitance relèvent des conditions technologiques et productives, et créent dans ces conditions une dépendance technologique du donneur d'ordres face au sous-traitant, la notion de dépendance mutuelle entretient la confusion sur la réalité des rapports de force qui se jouent dans les relations de travail. Le donneur d'ordres est le seul, en effet, à disposer du pouvoir de mobiliser du travail et d'engager ainsi le travail chez le sous-traitant. Cette capacité de mobilisation du travail crée non seulement de la dépendance économique, compte tenu du faible nombre de clients du sous-traitant, mais aussi le pouvoir d'évaluer le travail réalisé. La tendance que l'on constate actuellement est que les donneurs d'ordres gardent le pouvoir d'évaluer le résultat mais aussi celui de contrôler les moyens d'exécuter le travail par la mise en place d'une série d'indicateurs et de procédures et, par là, de prendre des décisions de gestion chez les soustraitants. La mise en place de ces indicateurs par les donneurs d'ordres les conduit à intervenir de façon quasi directe dans la gestion de la main-d'œuvre des sous-traitants ${ }^{14}$.

Cet état de dépendance a des conséquences sur la rentabilité des entreprises preneurs d'ordres. La moyenne des taux de profit se différencie ainsi nettement selon l'état de subordination des entreprises. En 2003, les preneurs d'ordres enregistraient, en moyenne individuelle, un taux de profit de $18,2 \%$, alors que celui des non-preneurs d'ordres était de $44,8 \%$. D'après nos calculs ${ }^{15}$, la présence d'un taux de profit plus faible accroît la probabilité d'être preneur d'ordres, confirmant ainsi l'hypothèse de dépendance économique vis-à-vis de leurs donneurs d'ordres. Cette profitabilité dégradée des preneurs d'ordres se traduit aussi en termes de conditions d'emploi et de travail de leurs salariés, qui bénéficient de salaires en moyenne moins élevés et qui subissent euxmêmes un éclatement de leur collectif, les preneurs d'ordres recourant davantage à la sous-traitance et à l'intérim ${ }^{16}$. Nos résultats indiquent que l'estimation de la probabilité d'être preneur d'ordres fait apparaître que la faiblesse du taux de profit a un lien propre avec le fait d'être preneur d'ordres, indépendamment de la taille de ces entreprises. En revanche, les éléments participant à la dégradation des conditions d'appartenance à un collectif protecteur se cumulent dans les entreprises preneurs d'ordres : être d'une taille inférieure à 50 salariés, voire à 100 salariés, augmente aussi la probabilité d'être preneur d'ordres. Le capital semble certes se hiérarchiser et se fragmenter mais, face au travail, il garde son unité : dans la lutte incessante qui oppose les capitalistes entre eux, le

14 R. Beaujolin, Les vertiges de l'emploi. L'entreprise face aux réductions d'effectifs, Paris, Grasset, 1999.

15 Voir tableau 1 en annexe pour les résultats de l'estimation des facteurs explicatifs du fait d'être preneur d'ordres exclusif (c'est-à-dire que la sous-traitance reçue représente plus de $97 \%$ du chiffre d'affaires du preneur d'ordres).

16 En effet, les résultats d'estimation présentés dans le tableau 1 en annexe indiquent que la probabilité d'être preneur d'ordres exclusif augmente quand l'entreprise recourt davantage à l'intérim (puisque le coefficient du taux d'intérim est positif et significatif dans la régression) et quand elle sous-traite davantage (puisque le coefficient du taux de sous-traitance est lui aussi positif et significatif). Ce dernier point illustre le principe de la sous-traitance en cascade : le preneur d'ordres exclusif (défini comme ayant la principale partie de son activité qui relève de la commande) fait faire une partie du travail qui lui a été commandé à une autre entreprise, et devient alors donneur d'ordres en plus d'être preneur d'ordres. 
preneur d'ordres est en quelque sorte créé par la demande qui émane du donneur d'ordres pour embaucher du travail dans de moins bonnes conditions et ainsi contourner les conquêtes salariales permises par l'État social. En effet, au début du siècle, le droit du travail a construit la protection du travailleur salarié sur la base du critère de subordination juridique, c'est-à-dire celui de la subordination personnelle du salarié aux ordres et directives de son employeur. Et l'État social a développé ses instruments de protection de l'emploi à partir de ce critère qui s'adaptait au régime de croissance fordiste. Le travail salarié dans le cadre des rapports de sous-traitance implique une tout autre définition de la dépendance : dépendance économique du salarié face au chômage de masse, dépendance économique du sous-traitant vis-à-vis du pouvoir de mobilisation du travail du donneur d'ordres, subordination «juridique » du salarié aux ordres et directives de son employeur. Il reste d'ailleurs un terme à inventer pour désigner la subordination du salarié aux ordres et directives d'un employeur de fait qui n'a pas de responsabilité en droit - retour de la force nue du capital.

Le fait que ce type de sous-traitance de substitution parvienne à se développer est l'expression même de la puissance du capital face au travail, lequel peut de moins en moins bien résister à mesure que l'on s'éloigne du plein-emploi. Le donneur d'ordres fait alors passer, sur le plan comptable, du capital variable pour du capital constant, du travail vivant pour du travail mort, une mobilisation de main-d'œuvre pour un contrat commercial : le contrat de travail disparaît pour le donneur d'ordres. Le travailleur salarié par le preneur d'ordres ne bénéficie pas de rémunération et de protections, héritées de l'État social, aussi bonnes que celles des travailleurs salariés directement par le donneur d'ordres, bien qu'il dépende réellement de ce dernier. Disposer effectivement d'une force de travail tout en se soustrayant au droit du travail, tel est le rôle de la sous-traitance. Mais cet effet d'évitement des règles ne se joue pas seulement par la substitution du contrat commercial au contrat de travail, il se déploie dans l'ensemble du tissu productif par la réduction de la taille des unités productives.

Cette réduction, que les économistes industriels nomment «désintégration verticale », et, en contrepartie, la montée des relations contractuelles plus ou moins élaborées entre les entreprises, recompositions auxquelles on assiste depuis une trentaine d'années, marquent un moment particulier de l'histoire récente du développement capitaliste. Il exprime l'une des stratégies employées par le capital pour contourner le New deal, auquel il n'avait pu se soustraire cinquante ans auparavant et qui lui servit même de béquille face à la déflation et à la menace communiste. Selon nos résultats, les entreprises industrielles qui sous-traitent le plus sont, toutes choses égales par ailleurs, celles qui ont le plus réduit leurs effectifs au cours des vingt dernières années, alors même qu'elles enregistraient une croissance de leur activité. La sous-traitance est donc associée à un processus de réduction de la taille des entreprises. Ainsi, déléguer des tâches à des sous-traitants pratiquant des salaires plus bas et des conditions de travail moins avantageuses peut aussi restreindre l'incidence du droit du travail pour les salariés du donneur d'ordres en raison des seuils d'effectifs instaurés par le droit du travail au cours de la période de plein-emploi ${ }^{17}$. En outre, le développement de la soustraitance est source de création d'entreprises preneurs d'ordres, pour lesquelles la part des entreprises dont l'effectif est inférieur à 50 salariés ${ }^{18}$ est significativement plus élevée que celle des non-preneurs d'ordres.

Mais cette diminution de la taille du travailleur collectif n'est que formelle. Elle est destinée à éviter les protections, elles aussi formelles, issues de la période précédente et à créer de nouvelles unités productives qui, d'une part, n'héritent pas d'accords d'entreprise ou de branche aussi avantageux et qui, d'autre part, bénéficient d'obligations légales moins contraignantes en raison même de leur taille.

17 C. Perraudin, N. Thèvenot, B. Tinel, J. Valentin, «Sous-traitance et ineffectivité du droit du travail : une analyse économique », op. cit.

18 Le seuil juridique de 50 salariés modifie les droits en matière d'hygiène et de sécurité, d'emploi (règlement du licenciement collectif) et surtout de représentation collective (obligation de créer un comité d'entreprise). 


\section{Sous-traitance, responsabilité de l'emploi et réinstitution de la subordination formelle}

Le travailleur collectif demeure et se voit donc en réalité recomposé à travers des unités indépendantes sur le plan comptable et/ou juridique. Ces transformations ont des effets bien réels : elles agissent à la baisse sur le salaire et sur les conditions de travail. Elles permettent en effet de se défaire plus facilement de la main-d'œuvre, puisque celle-ci est formellement considérée du point de vue du donneur d'ordres comme ne relevant pas du droit du travail ; le salarié en sous-traitance intériorise cette facilité de licencier pour le capital donneur d'ordres, par le truchement d'une rupture de contrat de sous-traitance ou d'une absence de renouvellement (tout ceci se résumant par la « contrainte du marché » que ne manquera pas d'invoquer le capital preneur d'ordres pour pressurer ou licencier effectivement sa force de travail), ce qui augmente le pouvoir de discipline du capital sur la main-d'œuvre et accroit donc encore davantage la subordination formelle. De ce point de vue, la sous-traitance représente une nouvelle modalité dans le processus de subordination. La responsabilité de l'emploi se trouve ici fortement questionnée dans la mesure où le donneur d'ordres n'entretient pas de lien juridique avec les travailleurs extériorisés, même s'il est responsable, dans les faits, des conditions d'emploi et de travail liées à l'état de subordination réelle dans lequel se trouvent les travailleurs extériorisés.

En rompant l'unité formelle du capital par l'introduction d'une discontinuité juridique entre donneur et preneur d'ordres, donc par les artifices permettant de dissimuler la mobilisation de la maind'œuvre, discontinuité qui n'existait pas à l'intérieur de la grande entreprise entre les différentes unités, la sous-traitance crée de toutes pièces une «contrainte de marché » destinée à produire une nouvelle discipline à l'encontre d'une main-d'œuvre déjà trop émancipée par l'État social : la soustraitance induit un retour au premier plan de la menace de rupture contractuelle, ce qui vient comme réinstituer la subordination formelle, ce moment primitif des rapports de production capitalistes. 
Annexe

\section{Graphique 1 : Augmentation du recours et de l'intensité du recours à la sous-traitance}

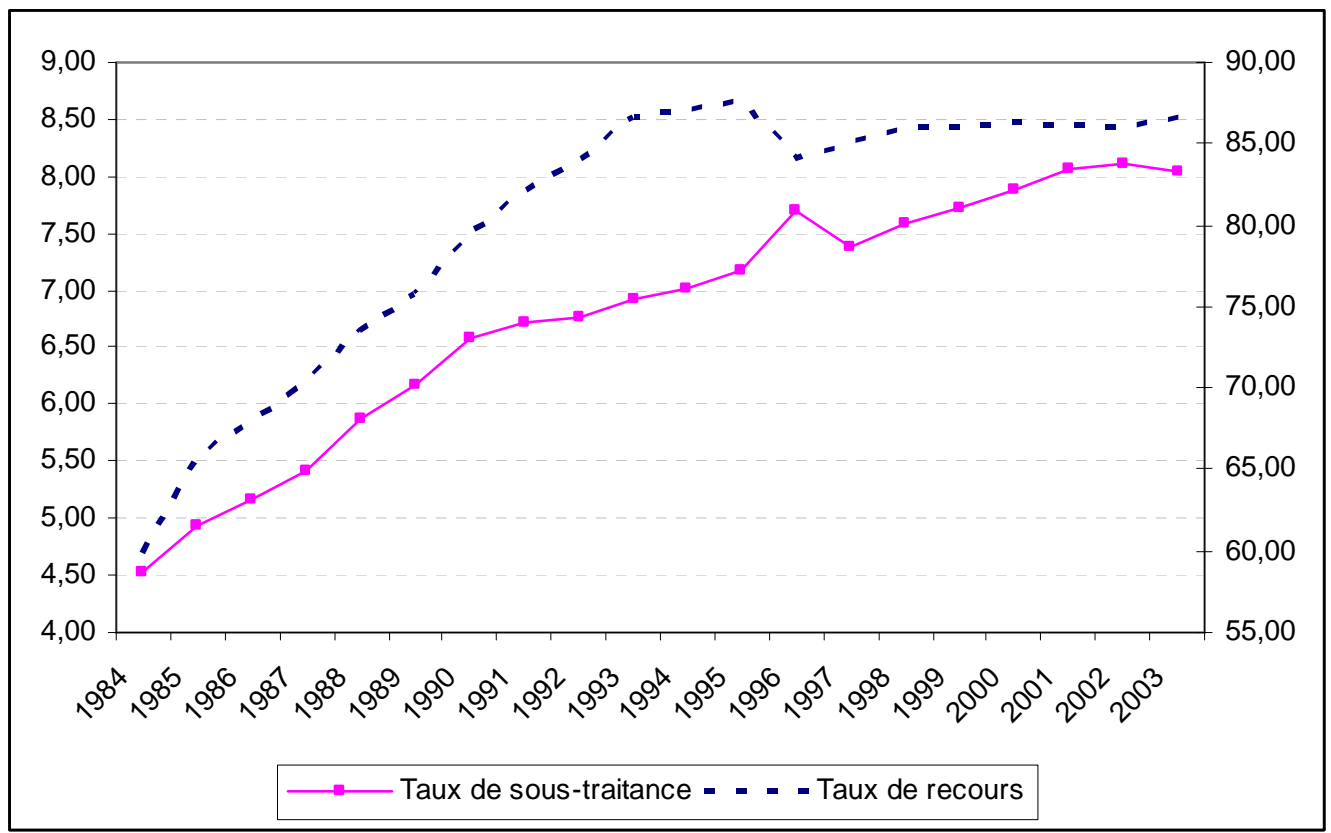

Source : Enquêtes Annuelles d'Entreprises, Calcul des auteurs.

Champ: Entreprises de 20 salariés et plus de l'Industrie des secteurs des biens de consommation, des biens intermédiaires et des biens d'équipement en France.

Lecture : Le taux de recours à la sous-traitance est passé de $60 \%$ des entreprises en 1984 à $87 \%$ en 2003 (échelle de droite). La moyenne des taux de sous-traitance individuels (dépenses de sous-traitance rapportées au chiffre d'affaires de l'entreprise) est passée de 4,5 en 1984 à 8 en 2003 (échelle de gauche).

\section{Graphique 2 : Part des dépenses de travail externes dans les dépenses de travail totales}

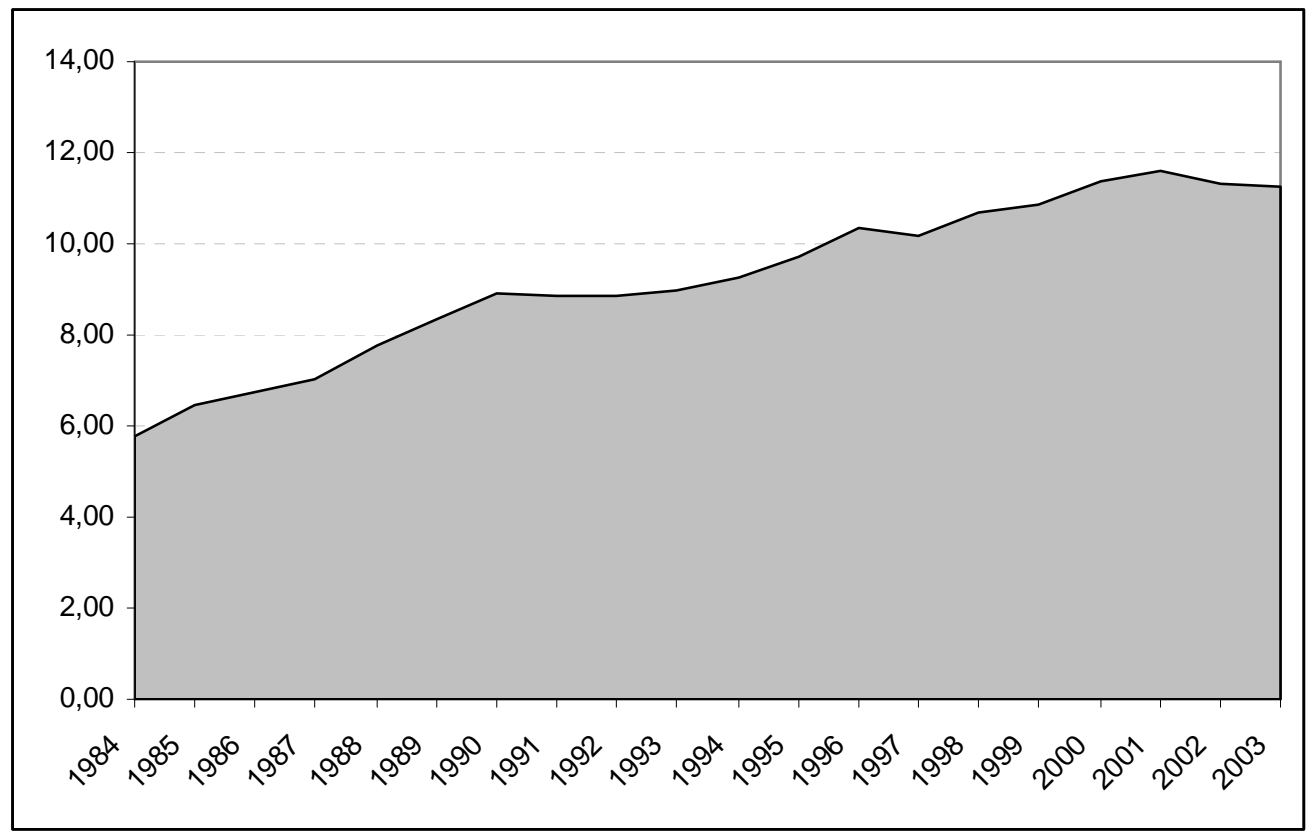

Source : Enquêtes Annuelles d'Entreprises, Calcul des auteurs.

Champ: Entreprises de 20 salariés et plus de l'Industrie des secteurs des biens de consommation, des biens intermédiaires et des biens d'équipement en France.

Lecture : La part des dépenses de travail externes (liées à la mobilisation de travail via la sous-traitance, mesurées par la moitié des dépenses de sous-traitance) représentait, en 1984, 5,8 \% des dépenses de travail totales (somme des salaires et cotisations versés aux salariés, et des dépenses de travail externes), alors qu'elles représentent 11,2\% en 2003. 
Tableau 1 : Facteurs explicatifs du fait d'être preneur d'ordres exclusif - 2003

\begin{tabular}{|l|c|c|}
\hline $\begin{array}{l}\text { Variable expliquée : probabilité d'être preneur } \\
\text { d'ordres exclusif }\end{array}$ & Coefficients & (écarts-types) \\
\hline Constante & 0.713 & $(0.1385)$ \\
Taille de l'entreprise & & \\
De 20 à 49 salariés & $0.433^{* * *}$ & $(0.0603)$ \\
De 5099 salariés & $0.248^{* * *}$ & $(0.0712)$ \\
De 100 à 250 salariés & Réf & \\
Plus de 250 & $-0.337^{* * *}$ & $(0.1013)$ \\
Secteur & & \\
Biens de consommation & $-1.007^{* * *}$ & $(0.0529)$ \\
Biens d'équipement & $-1.023^{* * *}$ & $(0.0522)$ \\
Biens intermédiaires & Réf & \\
Taux de profit & $-0.0005^{* * *}$ & $(0.000165)$ \\
Taux de sous-traitance & $0.059^{* * *}$ & $(0.00277)$ \\
Part des salaires dans la valeur ajoutée & $0.0019^{* * * *}$ & $(0.000599)$ \\
Salaire moyen & $-0.024^{* * *}$ & $(0.00233)$ \\
Taux d'intérim & $0.062^{* * *}$ & $(0.00213)$ \\
\hline
\end{tabular}

*** indique la significativité au seuil de $1 \%$.

Estimation Logit de la probabilité d'être preneur d'ordres exclusif (c'est-à-dire que la soustraitance reçue représente plus de $97 \%$ du chiffre d'affaires de l'entreprise).

Note de lecture : Le fait d'avoir entre 20 et 49 salariés, relativement au fait d'en avoir de 100 à 250 (référence), augmente la probabilité d'être preneur d'ordres exclusif (par rapport à ne pas être preneur d'ordre exclusif). Avoir un taux d'intérim plus élevé augmente la probabilité d'être preneur d'ordres exclusif (puisque le coefficient de la variable Taux d'intérim est positif et significatif).

Champ : Entreprise de l'Industrie en France de 20 salariés et plus.

Source : EAE, calculs des auteurs, in Thèvenot, Valentin (2005). 